\title{
Beruflicher Hautschutz muss vorankommen
}

— Eine Studie zur Handekzemprävention bei über 1.000 Metallarbeitern ergab klare Anhaltspunkte dafür, dass Hautschutz und Hautpflege zusammen den Hautbefund am deutlichsten verbesserten. Weniger effektiv war Hautschutz alleine, während keinerlei Maßnahmen zu einer Verschlechterung der Hautbefunde führten [Kütting $\mathrm{B}$ et al. $\mathrm{Br}$ ] Dermatol 2010; 162: 362-70]. Doch dabei blieb offen, wie viel und wie oft Hautschutzmittel angewendet werden müssen und ob ein gegen den Standard Natriumlaurylsulfat (SLS) geprüftes Mittel auch gegen andere wässrige Irritanzien zuverlässig wirkt. Ein Forschungsprojekt der Deutschen Gesetzlichen Unfallversicherung zeigte laut Prof. Dr. Peter Elsner, Direktor der Klinik für Hautkrankheiten der Universität Jena, bei Pflegekräften, dass im Mittel nur eine Menge von knapp $1 \mathrm{mg} / \mathrm{cm}^{2}$ Hautschutzmittel aufgetragen wird - „immerhin mehr als bei Sonnenschutzmitteln", konstatierte Elsner. Weiterführende Untersuchungen zeigten, dass der schützende Effekt dosisabhängig ist und somit bei mittlerer Dosis des Hautschutzprodukts immerhin noch ein begrenzter Effekt zu erwarten ist. Weitere Untersuchungen zeigten, dass je nach Produkt von der Schutzwirkung gegenüber SLS nicht automatisch auf den Schutz gegen andere irritierende wässrige Substanzen geschlossen werden kann.

Elsner forderte daher in Zukunft Hautschutzmittel mit Dosierempfehlungen, dazu Dosierhilfen und eine Kopplung der Dosieranweisung an Handschuhgrößen, (also z.B. „Handschuhgröße 9: Tragen Sie mindestens drei Hübe auf!"). Als Vision bezeichnete er eine Kennzeichnung von Hautschutzmitteln mit definiertem Wirkin-

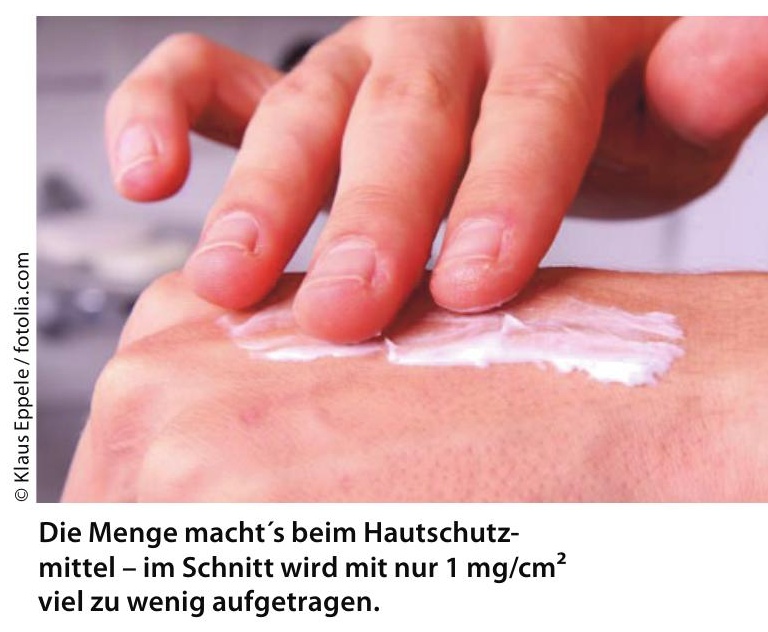

dex gegen ein bestimmtes Irritans. Für ihn steht fest: Hautschutzmittel sind keine Kosmetika. Folgerichtig forderte er für die Zukunft einen gesetzlichen Wirksamkeitsnachweis. Die Basis haben die Untersuchungen gelegt. Nun ist die Politik gefordert.

Friederike Klein

Elsner P. Hautschutz in der beruflichen Prävention. Symposium „Berufsdermatologie“

\section{Epikutantest gerettet}

— Seit 2004 gab es aufgrund der gestiegenen Anforderungen des Deutschen Arzneimittelgesetzes an die Zulassung keine neuen kommerziellen Kontaktallergene zur Testung mehr auf dem Markt. Begründet wurde dies mit den Regularien des europäischen Arzneimittelgesetztes - jetzt war scheinbar alles nur ein großes Missverständnis.

Deutsche Dermatologen haben in Publikationen, aber auch ganz persönlich ihren Einfluss in Sachen Kontaktallergenzulassung und Epikutantestung auf europäischer Ebene geltend gemacht: Mit Erfolg, wie Prof. Dr. Swen Malte John von der Abteilung Dermatologie, Universität Osnabrück, berichten konnte. Die Europäische Kommission konstatierte, dass die diagnostische Patchtestung nicht unter die Regelungen des europäischen Arzneimittelgesetzes falle und das Testmaterial nur ein Provokationsagens darstelle, kein auf Wirksamkeit oder Sicherheit zu prüfendes Arzneimittel. Entsprechend gelten auch nicht dieselben Anforderungen an epidemiologische Studien zur Zulassung der Kontaktallergene.

Daraufhin wurde im Juli 2012 mit dem Bundesministerium für Gesundheit ein Vorgehen vereinbart, dass "die Kalamität umrundet", wie John sich ausdrückte. Denn nach dem deutschen Arzneimittelgesetz gelten Kon-

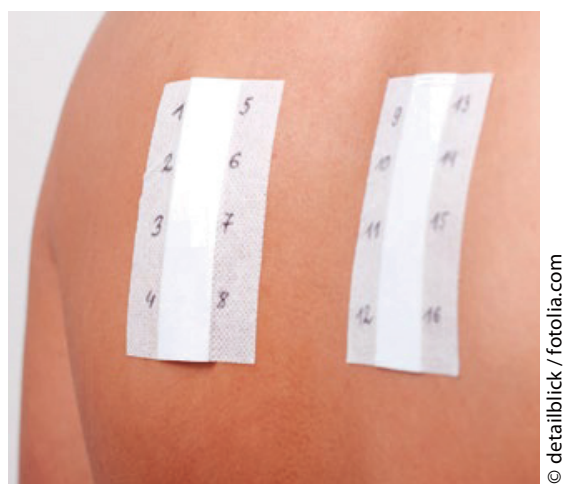

Für Allergene zur Epikutantestung gibt es jetzt ein vereinfachtes Zulassungsverfahren.

taktallergene weiterhin als Arzneimittel. Wichtig sei dabei gewesen, dass auch die gesetzliche Unfallversicherung an Bord war. Er ist optimistisch, dass es mit dem vereinbarten vereinfachten Zulassungsverfahren, das zum Beispiel nur eine begrenzte Patientenzahl erfordert, wieder möglich sein werde, neue Allergene zu identifizieren und in das Spektrum kommerzieller Testsubstanzen zu integrieren. Die Hersteller jedenfalls haben signalisiert, dass sie das vereinbarte Vorgehen mittragen können. Friederike Klein

John SM. Bedeutung für die Prävention und das therapeutische Vorgehen in der Praxis. Symposium „Berufsdermatologie"

\section{Personalisierte Ekzemtherapie}

— Einen Ausblick auf die zukünftigen Konzepte bei der atopischen Dermatitis gab Prof. Dr. Thomas Bieber, Universitätsklinik Bonn. Die Leitlinien sehen derzeit ein einheitliches Vorgehen für alle Patienten vor. Dies entspreche dem Grundsatz "One size fits all“. Die Arzneimittelindustrie, so Bieber, sei immer noch der Meinung, man könne durch ein einziges Präparat alle Patienten mit atopischer Dermatitis gleichsam behandeln. Für die Zukunft ist jedoch ein Umdenken notwendig. Den Begriff "stratifizierte Medizin“ erklärte er am Beispiel der Melanomtherapie. Hier ist nicht die klinische, sondern die molekulare Einteilung der Tumoren, etwa mit dem Vorliegen einer BRAF-Mutation, entscheidend für die Therapie. Bieber geht davon aus, dass auch das sehr heterogene Krankheitsbild der atopischen Dermatitis sich in Subformen entsprechend verschiedene Biomarker aufsplitten lässt. In Zukunft könnten biologische Marker wie etwa Mutationen des Filaggrin-Gens oder bestimmte Zytokin-Profile die Möglichkeit bieten, von frühester Kindheit an differenziert Prävention zu betreiben und zu therapieren.

Dr. Andreas Fischer

Bieber T. Atopische Dermatitis: Quo vadis?; Symposium "Atopische Dermatitis" 